\title{
Correction to: $\mathrm{O}_{2}$ permeability of lipid bilayers is low, but increases with membrane cholesterol
}

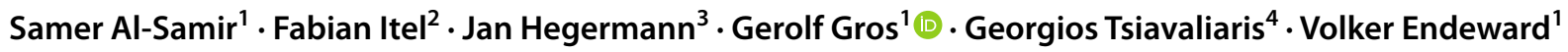

Accepted: 6 December 2021 / Published online: 31 January 2022

(c) Springer Nature Switzerland AG 2022

\section{Correction to:}

Cellular and Molecular Life Sciences

(2021) 78:7649-7662

https://doi.org/10.1007/s00018-021-03974-9

In the published original article, citation of reference 25 has been processed incorrectly.

Now this has been corrected. The original article has been updated.

Publisher's Note Springer Nature remains neutral with regard to jurisdictional claims in published maps and institutional affiliations.

The original article can be found online at https://doi.org/10.1007/ s00018-021-03974-9.

\section{Gerolf Gros}

Gros.Gerolf@MH-Hannover.de

1 AG Vegetative Physiologie 4220, Zentrum Physiologie, Medizinische Hochschule Hannover, 30625 Hannover, Germany

2 Empa, Swiss Federal Laboratories for Materials Science and Technology, Lerchenfeldstr. 5, CH-9014 St. Gallen, Switzerland

3 Abteilung Funktionelle und Angewandte Anatomie, Elektronenmikroskopie 8840, Medizinische Hochschule Hannover, 30625 Hannover, Germany

4 Abteilung Biophysikalische Chemie 4350, Medizinische Hochschule Hannover, 30625 Hannover, Germany 